\title{
ECOLOGÍA DE LA GERMINACIÓN EN ESPECIES DEL GÉNERO VERONICA (SCROPHULARIACEAE) DEL SO DE ESPAÑA
}

\author{
Rocío JUAN, Inmaculada FERNÁNDEZ y Julio E. PASTOR
}

RESUMEN. Ecología de la germinación en especies del género Veronica (Scrophulariaceae) del SO de España. Se ha realizado un estudio de germinación y viabilidad con semillas de 11 especies de Veronica del SO de España. En las pruebas de laboratorio, a $22 \pm 2{ }^{\circ} \mathrm{C}$, la respuesta de germinación fue distinta en luz y obscuridad. En luz, V. anagallis-aquatica, V. anagalloides, V. arvensis, V. persica y V. polita alcanzaron porcentajes de germinación altos (superiores al $80 \%$ ), mientras que en otras como $V$. cymbalaria, $V$. hederifolia, V. peregrina, V. praecox, V. scutellata y V. triphyllos, fueron bajos (inferiores al 20\%). En obscuridad, V. cymbalaria, $V$. hederifolia y $V$. peregrina experimentaron un aumento significativo de dicho porcentaje. La viabilidad de las semillas fue muy alta para todas las especies, variando entre el $81 \%$ y el $100 \%$. Se discuten los requerimientos para la germinación de cada especie en relación a su hábitat natural.

Palabras clave. Germinación, dormición, luz, semillas, Veronica, Scrophulariaceae, SO de España.

ABSTRACT. Seed germination ecology of Veronica species (Scrophulariaceae) from SW Spain. Seed germination and viability were studied in several species of Veronica. Laboratory germination experiments at $22 \pm 2{ }^{\circ} \mathrm{C}$ showed a different response in light and the dark. In light, V. anagallis-aquatica, $V$. anagalloides, $V$. arvensis, $V$. persica and $V$. polita achieved high germination rates (greater than $80 \%$ ), while others such as $V$. cymbalaria, $V$. hederifolia, V. peregrina, V. praecox, V. scutellata and $V$. triphyllos significantly reduced seed germination (lower than 20\%). Seed germination percentage of $V$. cymbalaria, $V$. hederifolia and $V$. peregrina increased significantly in the dark. Seed viability was very high by all species (from $81 \%$ to $100 \%$ ). The relatinships between seed germination requeriments and species habitat is discussed.

Key words. Germination, dormancy, light, seeds, Veronica, Scrophulariaceae, SW Spain.

\section{INTRODUCCIÓN}

Las especies del género Veronica, del SO de España, se presentan en hábitats muy diferentes como pueden ser: tierras de cultivos, donde abundan generalmente especies anuales ( $V$. hederifolia L., $V$. persica Poiret, $V$. triphyllos L., V. polita Fries y V. arvensis L.), pedregales en su mayoría calizos ( $V$. cymbalaria Bodard), zonas altas de montaña (V. praecox L.), jardines (V. peregrina L.) y hasta lugares encharcados o bordes de arroyos donde se localizan, además de $V$. anagalloides Guss., los únicos taxones perennes tratados en 
este estudio, $V$. anagallis-aquatica L. y $V$. scutellata L.

Este género contiene especies que forman muchas semillas pequeñas, y otras que producen pocas semillas y grandes, lo que induce a pensar que pudieran presentar distintos patrones de germinación. Harris \& Lovell (1980) y Baskin \& Baskin (1983) ya manifestaron que las especies de Veronica difieren en sus estrategias reproductivas, requerimientos de germinación y forma de crecimiento.

Las semillas viables que no germinan bajo unas condiciones ambientales normales deben considerarse durmientes o latentes. De los cinco tipos de latencia establecidos por Baskin \& Baskin (1989), la latencia fisiológica es la que aparece más frecuentemente entre las especies anuales de invierno, es decir, aquellas que germinan en otoño como ocurre en algunas especies del género Veronica (Baskin \& Baskin, 1983), además es el tipo de latencia que presentan la mayoría de las semillas que entran a formar parte del banco de semillas de los suelos en zonas templadas (Baskin \& Baskin, 1989). Éstas pueden permanecer en el suelo un tiempo variable, así Oostin \& Humphreys (1940) encontraron en Carolina del Norte semillas viables de $V$. arvensis de hasta 85 años, por lo que estas especies pueden tener un papel importante en la composición de los bancos de semillas.

La razón principal de que las semillas no germinen es porque requieren luz para ello (Baskin \& Baskin, 1984). Sin embargo, (Harris \& Lovell, 1980; Baskin \& Baskin, 1983, etc.) demostraron que algunas especies requieren condiciones de obscuridad para germinar. Por otra parte, Janssen (1973) indica que además de la iluminación, otro factor muy importante es la temperatura, sobre todo en las especies denominadas anuales de invierno.

En este estudio se quiere determinar la capacidad de germinación de las semillas de 11 especies de Veronica, en respuesta a la modificación de algunas condiciones ambientales. Se examinan velocidad y porcentaje de germinación, así como la viabilidad, expresada en tanto por ciento para cada especie.

\section{MATERIAL Y MÉTODOS}

Se han utilizado semillas maduras de 11 especies de Veronica recolectadas entre los meses de Marzo y Junio de dos temporadas sucesivas (1992 y 1993). Estas semillas fueron almacenadas en tubos herméticos de plástico con gel de sílice, en una caja a temperatura ambiente. Para las pruebas de germinación, siempre que fue posible, se emplearon tres poblaciones por especie, utilizand o 50 semillas por población para cada experimento. Las semillas se colocaron en placas de Petri, sobre una lámina de papel de filtro, con humedad constante, entre los meses de Octubre a Enero del mismo año de recolección. En primer lugar, todas las placas se pusieron en las mismas condiciones en un laboratorio con luz artificial (46 lx) durante 12 horas aproximadamente y a una temperatura de $22 \pm 2{ }^{\circ} \mathrm{C}$ durante 30 días. Las placas se controlaron diariamente, retirando las semillas germinadas. Aquellas especies que no germinaron en estas condiciones o dieron un porcentaje inferior al $20 \%$, volvieron a sembrarse posteriormente, con la misma temperatura pero sometiéndolas a total obscuridad durante 30 días, sistema ya utilizado anteriormente en Veronica (Harris \& Lovell, 1980; Baskin \& Baskin, 1983). Por último, la única especie que presentó en ambos casos un $0 \%$ de germinación se puso durante 14 días a $4{ }^{\circ} \mathrm{C}$, para sembrarlas posteriormente a $22 \pm 2{ }^{\circ} \mathrm{C}$ tanto en luz como en obscuridad (Harris \& Lovell, 1980).

La viabilidad de las semillas no germinadas se ha determinado al final del proceso, diseccionándolas bajo un microscopio estereóscopico y considerándolas viables cuando contenían embrión (Baskin \& Baskin, 
1981 y 1983; Baskin et al., 1986).

Cuando fue posible los datos se analizaron mediante el test de la «t de Student» de comparación de medias. En aquellos casos en que el número de poblaciones, y por tanto de placas, fue pequeño se usó el test de comparación de proporciones de la «chicuadrado» (Sokal \& Rohlf, 1979).

El origen de las poblaciones estudiadas se indica en el Apéndice.

\section{RESULTADOS}

En las condiciones de iluminación y temperatura establecidas, se han alcanzado porcentajes de germinación muy variables entre las 11 especies estudiadas (tab. 1). Dichos porcentajes permiten separar claramente dos grupos de especies. Por un lado, V. polita, $V$. persica, V. arvensis, $V$. anagallis-aquatica y $V$. anagalloides que presentan un porcentaje de germinación superior al $80 \%$; y por otro lado el resto (V. cymbalaria, V. peregrina, $V$. hederifolia, $V$. praecox, $V$. scutellata y $V$. triphyllos) cuyo porcentaje de germinación no superó el 20\%. Cuando se compararon ambos grupos mostraron diferencias significativas ( $p<0.05$ para todas las comparaciones), mientras que las comparaciones realizadas dentro de cada grupo no mostraron tales diferencias ( $>0.05$ para todas las comparaciones).

Las especies de este último grupo con porcentajes bajos de germinación, se volvieron a sembrar a igual temperatura pero en obscuridad (tab. 1). Como puede apreciarse en tres de ellas (V. peregrina, V. cymbalaria y $V$. hederifolia) el porcentaje de germinación aumentó significativamente al 24, 83 y $42 \%$ respectivamente $(\mathrm{p}<0.05$ para $V$. peregrina y $\mathrm{p}<0.01$ para las otras dos especies), mientras que en las restantes no se apreciaron estas diferencias.

En V. triphyllos tampoco se observó cambios en su respuesta de germinación después de 14 días a $4{ }^{\circ} \mathrm{C}$.

De las disecciones realizadas después de los test de germinación se obtuvo que para nueve de las especies estudiadas la viabilidad fue cercana al $100 \%$ (tab. 1), sólo V. polita y $V$. peregrina presentaron un porcentaje algo

\begin{tabular}{lcrrr}
\hline Especie & \multirow{2}{*}{$N^{\circ}$ Pob. } & \multicolumn{2}{c}{ Germ. $(\%)$} & Viab. Sem.(\%) \\
& & Luz & Obsc. & \\
\hline V. anagallis-aquatica & 3 & 98 & -- & 100 \\
V. anagalloides & 1 & 100 & -- & 100 \\
V. arvensis & 3 & 97 & -- & 98 \\
V. cymbalaria & 3 & 19 & $83 * *$ & 100 \\
V. hederifolia & 3 & 0 & $42 * *$ & 100 \\
V. peregrina & 2 & 7 & $24 *$ & 81 \\
V. persica & 3 & 98 & -- & 94 \\
V. polita & 3 & 92 & -- & 90 \\
V. praecox & 1 & 9 & $8 \mathrm{~ns}$ & 100 \\
V. scutellata & 1 & 18 & $12 \mathrm{~ns}$ & 99 \\
V. triphyllos & 3 & 0 & $0 \mathrm{~ns}$ & \\
\hline
\end{tabular}

Tabla 1. Número de poblaciones, porcentajes de germinación de las semillas viables en luz y obscuridad, y porcentaje de viabilidad de las semillas. (Chi-cuadrado de comparación de porcentajes; ns = no significativo, $* \mathrm{p}<0.05, * * \mathrm{p}<0.01)$. Number of populations, germination percentages of viable seeds in light and dark, and viability seed percentages of Veronica species. (Chi-square; ns = not significant, * $p<0.05$; ** $p<0.01$ ). 
inferior (90 y $81 \%$, respectivamente). Al diseccionar las semillas, se ha comprobado que éstas siempre están totalmente embebidas, por lo que no cabe esperar que alguna de las especies estudiadas presenten latencia mecánica.

Respecto a la velocidad de germinación obtenida en condiciones de iluminación, $V$. anagalloides es la que lo hace con mayor rapidez, ya que comienza a germinar al tercer día y al sexto ha alcanzado el 100\%. También presentan una velocidad alta de germinación, $V$. polita, V. persica, V. anagallis-aquatica y $V$. arvensis, pues empiezan al tercer o cuarto día y alcanzan sus mayores porcentajes entre el séptimo y undécimo día (Fig. 1). Sin embargo, $V$. peregrina, V. scutellata, $V$. praecox y $V$. cymbalaria, germinan más tarde ya que no comienzan hasta el sexto o séptimo día, presentando posteriormente incrementos muy bajos salvo en $V$. cymbalaria en la que los mayores incrementos se alcazan entre el duodécimo y decimocuarto día, aunque en ningún caso alcanzan porcentajes superiores al $20 \%$.

En cuanto a las especies colocadas en obscuridad, como puede observarse en la Fig. 2, V. cymbalaria fue la más rápida en germinar, seguida de $V$. hederifolia, $V$. scutellata y $V$. praecox. Pero quizás lo más llamativo de este conjunto de especies es que el inicio de la germinación en obscuridad y luminosidad es semejante, lo que conlleva a decir que en ambas condiciones la germinación se encuentra retrasada con respecto al grupo de $V$. polita.

\section{DISCUSIÓN}

La mayoría de las especies del género Veronica, en condiciones naturales, germinan durante el otoño, presentando semillas maduras al inicio de la primavera, las especies más efímeras, y a finales de la primavera o comienzo del verano, las de ciclo más largo. Dichas semillas permanecerán en el suelo durante todo el verano, donde dependiendo del área se alcanzarán temperaturas superiores a los 50 ${ }^{\circ} \mathrm{C}$. Entre las especies denominadas anuales de invierno, hay que distinguir las obligadas y las facultativas (Baskin \& Baskin, 1989). Las anuales de invierno obligadas presentan un ciclo anual de latencia/no latencia (Baskin \& Baskin, 1984), mientras que las facultativas tienen un ciclo anual de latencia condicional/ no latencia (Baskin \& Baskin, 1981). En las especies de Veronica estudiadas pueden observarse ambos comportamientos.

A partir de los resultados obtenidos se puede decir que el tipo de latencia que presentan las especies del género Veronica, tanto anuales como perennes cuando existe, es fisiológica (Baskin \& Baskin, 1989) ya que las semillas no germinadas son permeables al agua y el embrión está completamente desarrollado.

Entre las especies perennes y anuales no se puede establecer ninguna diferencia de comportamiento basada en los resultados (tab. 1). No obstante, las dos especies perennes estudiadas, $V$. anagallis-aquatica y $V$. scutellata se comportan de forma distinta, presentando un 98 y un $18 \%$ de germinación respectivamente (en condiciones de iluminación). Así, V. anagallis-aquatica parece comportarse como una especie perenne típica sin que las semillas presenten cambios de latencia una vez que éstas dejan de estar durmientes, como ocurre en Potentilla recta (Baskin \& Baskin, 1990) y Portulaca smalii (Baskin et al., 1987). Sin embargo, V. scutellata parece tener un comportamiento más complejo, similar al de una anual de invierno facultativa, también observado en otras Scrophulariaceae perennes como Penstemon palmeri (Meyer \& Kitchen, 1992); así, cuando las condiciones son desfavorables, las semillas entran en una latencia condicional y necesitarán un periodo prolongado de condiciones favorables para germinar (Allen \& Meyer, 1990). La existencia de un verdadero ciclo de latencia no se ha 


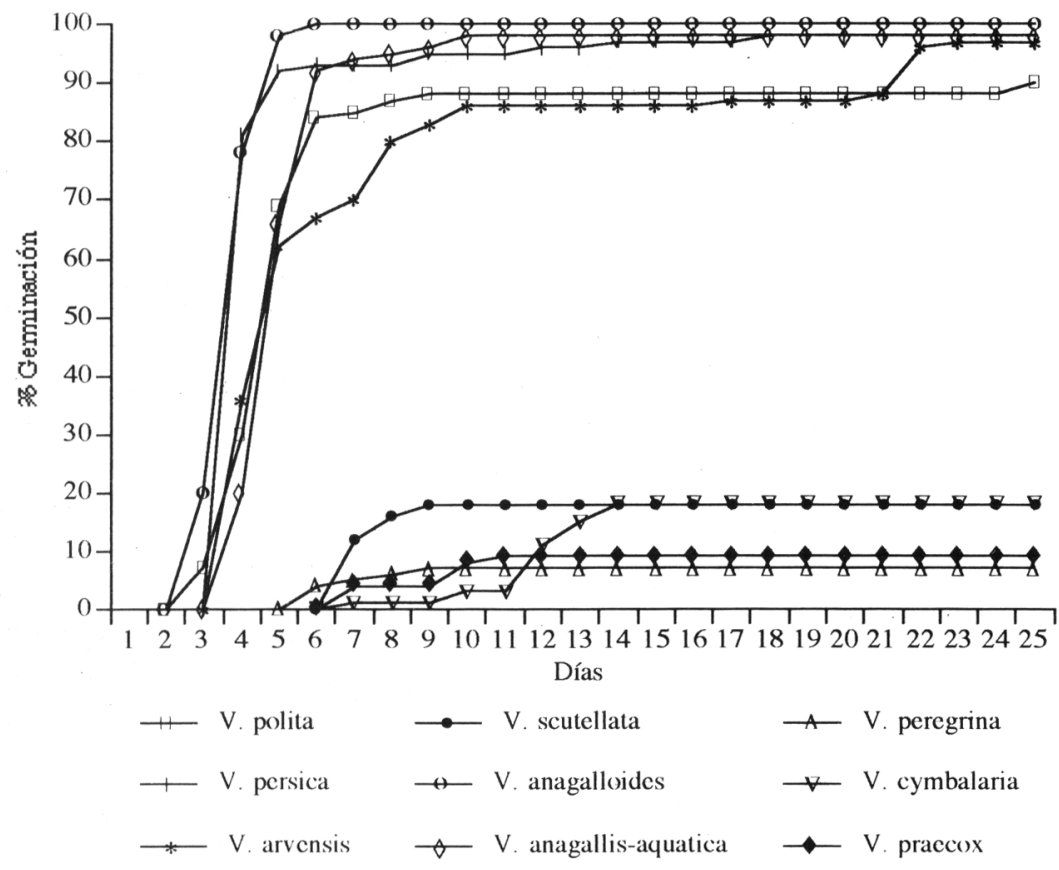

Figura 1. Curva de germinación de 9 de las especies de Veronica estudiadas en condiciones de iluminación. Germination curve of 9 species of Veronica in light conditions.

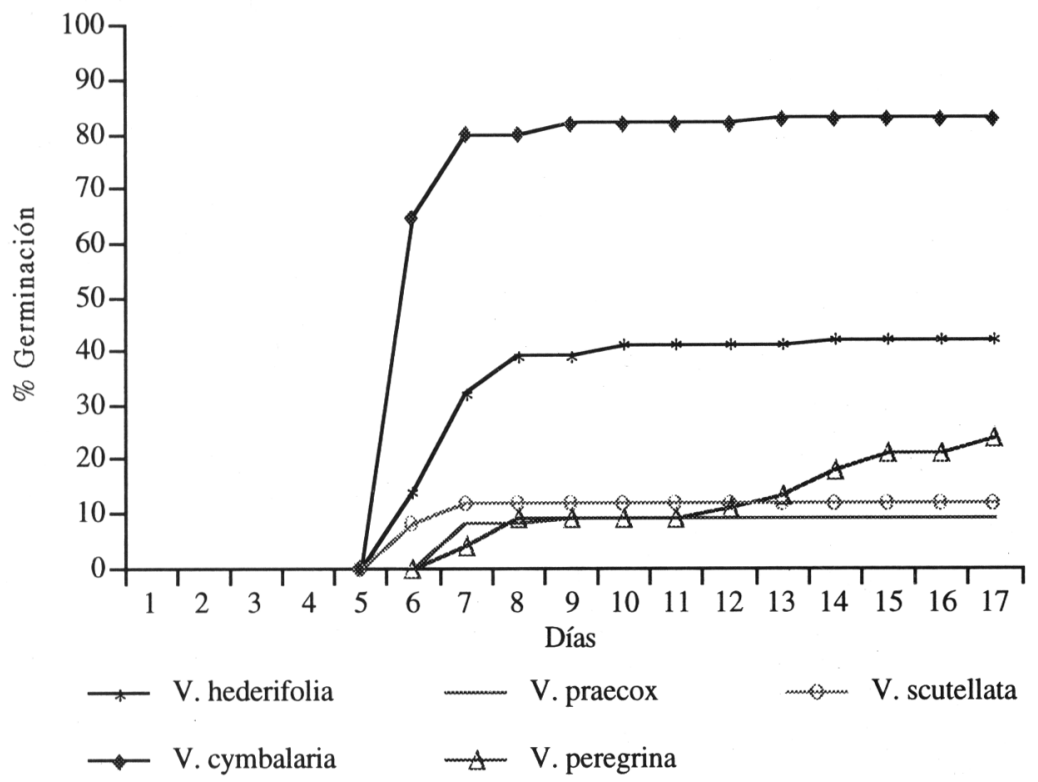

Figura 2. Curva de germinación de las 5 especies de Veronica estudiadas en condiciones de obscuridad. Germination curve of 5 species of Veronica in dark conditions. 
demostrado en especies perennes, pero parece que especies de regiones templadas adaptan la necesidad de germinar a una estación, manteniendo así un banco permanente de semillas (Meyer \& Kitchen, 1992). Respecto al grado de iluminación, si se tiene en cuenta que $V$. scutellata crece semicubierta por la vegetación alta de marisma (juncales), donde las condiciones de luz son escasas, cabría esperar un aumento de la germinación en obscuridad (Harris \& Lovell, 1980), sin embargo, los porcentajes de germinación obtenidos en ausencia y presencia de luz para $V$. scutellata no muestran diferencias significativas (tab. 1), por lo que deben ser otros factores los responsables del alto porcentaje de semillas viables que no germinan, pasando a formar parte del banco de semillas.

Respecto a las especies anuales se observaron también diferencias en el comportamiento (tab. 1). No obstante, Harris \& Lovell (1980) comprobaron que todas las especies de Veronica tratadas, excepto $V$. hederifolia, germinaban bien en condiciones de buena iluminación $(4 \mathrm{Klx})$ y a $24{ }^{\circ} \mathrm{C}$. Además, observaron una correlación entre los requerimientos lumínicos de las semillas y su tamaño, ya que las especies con semillas pequeñas reducían la germinación en obscuridad ( $V$. arvensis) o no germinaban ( $V$. serpyllifolia). Teniendo en cuenta el tamaño de las semillas de estas especies (Juan et al., 1994) es difícil establecer relaciones entre el porcentaje de germinación en condiciones de buena iluminación y el tamaño de sus semillas, pues aunque en líneas generales las especies con semillas pequeñas tienen un porcentaje alto de germinación ( $V$. arvensis, $V$. anagalloides), y las de semillas grandes más bajo ( $V$. cymbalaria, $V$. hederifolia), se observaron algunas excepciones ya que $V$. peregrina con semillas pequeñas sólo mostró un $7 \%$ de germinación, mientras que $V$. polita y $V$. persica que tienen semillas relativamente grandes germinaron bien $(90$ y $94 \%$ respectivamente) (tab. 1). De igual forma, $V$. peregrina que tiene semillas pequeñas presentó un aumento considerable en el porcentaje de germinación cuando se sometió a total obscuridad, en contraposición a lo que cabría esperar según Harris \& Lovell (1980). Posiblemente, la mayoría de estas especies se comportan como anuales de invierno obligadas, necesitando el transcurso del verano para germinar en el otoño. Por ello, estas especies sólo aparecen en el campo durante la primavera. Excepcionalmente, $V$. persica se pudo recolectar tanto en primavera como en otoño, mostrando así un ciclo normal de una anual de invierno facultativa, las cuales germinan en otoño pero también pueden hacerlo en primavera si las condiciones son favorables. Igualmente, Baskin \& Baskin (1983), en su estudio sobre $V$. arvensis observaron que aunque la mayoría de las semillas germinaban en otoño, había algunas que podían hacerlo en primavera.

Entre las especies sembradas en obscuridad, $V$. hederifolia y $V$. cymbalaria fueron las que presentaron incrementos más notables en el porcentaje de germinación. Así, $V$. hederifolia pasó del $0 \%$ de germinación al $42 \%$. Este hecho, además de estar de acuerdo con la correlación establecida por Harris \& Lovell (1980), indica que en condiciones normales sus semillas han de estar enterradas para germinar. Teniendo en cuenta el hábitat de esta especie, que suele presentarse en suelos de cultivos removidos cada año, es fácil que las semillas queden enterradas favoreciendo la germinación. Es frecuente encontrar especies arvenses que no germinan con luz, pues si todas las semillas se volvieran no durmientes con luz y bajo condiciones de humedad favorables para la germinación, no habría bancos permanentes de semillas (Meyer \& Kitchen, 1992). El aumento de germinación de V. cymbalaria (del 19 al 83\%) resulta lógico si se tiene en cuenta que son propias de roquedos, y aunque éstos estén en laderas muy soleadas, 
al madurar las cápsulas dejan caer las semillas entre las grietas de las rocas donde las condiciones son más óptimas para la germinación. Esto está apoyado en observaciones de campo, donde se ha comprobado que la mayor parte de las plántulas de esta especie crecen entre las grietas más umbrías e incluso debajo de las piedras en canchales, siendo muy pocas las ocasiones en las que se han visto plántulas en lugares abiertos.

La única especie que no ha respondido a ninguno de los tratamientos, incluído una exposición a $4{ }^{\circ} \mathrm{C}$ durante 14 días, ha sido $V$. triphyllos. El área que presenta esta especie en el SO de España (Los Pedroches) hace pensar que sus semillas necesitan alcanzar en el suelo temperaturas muy elevadas durante el verano que podrían ser indispensables para su posterior germinación. El efecto de las altas temperaturas del verano hacen que algunas especies anuales de invierno obligadas pierdan su latencia para germinar en otoño (Baskin \& Baskin, 1983). Dicho efecto es especialmente notable en semillas de testa dura, como indicó Quinlivan (1961) en sus observaciones de algunas leguminosas, cuya testa se agrietaba por la fluctuación diaria de la temperatura.

\section{APÉNDICE}

V. polita:

HUELVA: Aracena, 14.3.92, Juan \& López (SEV 135140); SEVILLA: Coria del Río, 27.3.92, Juan (SEV 135098); Sevilla: 24.3.92, Arista \& Juan (SEV 135139).

\section{V. persica:}

CÁDIZ: Puerto de Santa María, 21.3.93, Arista (SEV 135102); HUELVA: Fuenteheridos, 14.3.92, Juan \& López (SEV 135104); SEVILLA: Coria del Río, 27.3.92, Juan (SEV 135141).

V. arvensis:

CÓRDOBA: Pozoblanco, La Romana, 6.4.92, Fernández \& Juan (SEV 135089); Villanueva del Duque, 6.4.92, Fernández \& Juan (SEV 135143);
SEVILLA: Sevilla, 25.3.92, Nieto (SEV 135142).

V. scutellata:

HUELVA: Doñana, 15.6.92, Díez, Fernández \& Juan (SEV 135076).

V. cymbalaria:

CÁDIZ: Entre San Roque y La Almoraima, 27.4.92, Arista \& Juan (SEV 135107); CÓRDOBA: Santa María de Trassierra, 1.4.93, Juan \& Pastor (SEV 135110); SEVILLA: Estepa, Pico Becerrero, 11.4.92, Juan \& Rodriguez (SEV 135111).

\section{V. anagalloides:}

CÓRDOBA: Entre Los Blázquez y Peraleda del Zaucejo, 9.6.92, Juan \& Pastor (SEV 135082).

\section{V. anagallis-aquatica:}

HUELVA: Arroyo de la Plata, 3.6.92, Arista \& Díez (SEV 135080); Candón, 7.6.93, Fernández \& Juan (SEV 135078); Río Tinto, 5.6.92, Juan, Pastor \& Santa-Bárbara (SEV 135077).

\section{V. triphyllos:}

CÓRDOBA: Belalcázar, 6.4.92, Fernández \& Juan (SEV 135084); Pozoblanco, 6.4.92, Fernández \& Juan (SEV 135083); Villanueva del Duque, 6.4.92, Fernández \& Juan (SEV 135135).

\section{$V$. hederifolia:}

CÓRDOBA: Carcabuey, 17.3.93, Fernández \& Juan (SEV 135115); HUELVA: Peña de Arias Montano, 6.4.93, Juan (SEV 135116); SEVILLA: Salteras, 14.4.93, Fernández \& Juan (SEV 135118).

\section{V. praecox:}

CÓRDOBA: Sierra de la Horconera, Pico Morrión, 1400 m.s.m., 29.5.93, Juan \& López (SEV 135085).

\section{V. peregrina:}

SEVILLA,: Coria del Río, 27.3.92, Juan (SEV 135093); Sevilla, 5.4.92, Santa-Bárbara (SEV 135094).

\section{BIBLIOGRAFÍA}

ALLEN, P.S. \& S.E. MEYER -1990- Temperature requeriments for seed germination of three 
Penstemon species. HortScience, 25(2): 191193.

BASKIN, J.M. \& C.C. BASKIN -1981- Seasonal changes in the germination responses of buried Lamium amplexicaule seeds. Weed Res., 21: 299-306

BASKIN, J.M. \& C.C. BASKIN -1983-Germination ecology of Veronica arvensis. J. Ecol., 71: 5768.

BASKIN, J.M. \& C.C. BASKIN -1984- Role of temperature in regulating timing of germination in soil seed reserves of Lamium purpureum $L$. Weed Res., 24: 341-349.

BASKIN, J.M. \& C.C. BASKIN -1989- Physiology of dormancy and germination in relatio to seed Bank Ecology. In M.A. LECK, V.T. PARKER \& R.L. SIMPSON (eds.) Ecology of soil seed Banks. Academic Press, Inc. 1989: 53-66.

BASKIN, J.M. \& C.C. BASKIN -1990- Role of temperature and light in the germination ecology of buried seeds of Potentilla recta. Ann. appl. Biol., 117: 611-616.

BASKIN, J.M., C.C. BASKIN \& J.C. PARR -1986Field emergence of Lamium amplexicaule $\mathrm{L}$. and Lamium purpureum $\mathrm{L}$. in relation to the annual seed dormancy cycle. Weed Res., 26: 185-190.

BASKIN, J.M., C.C. BASKIN \& J.F. McCORMICK -1987- Seasonal changes in germination response of buried seeds of Portulaca smalii. Bull. Torrey Bot. Club, 114: 169-172.

HARRIS, G.R. \& P.H. LOVELL -1980- Growth and reproductive strategy in Veronica spp. Ann. Bot., 45: 447-458.

JANSSEN, J.G. -1973-Effects of light, temperatura and seed age on the germination of the winter annuals Veronica arvensis L. and Myosotis ramosissima Rochel ex Schult. Oecologia, 12: 141-146.

JUAN, R., J. PASTOR \& I. FERNANDEZ - $1994-$ Seed morphology in Veronica L. (Scrophulariaceae) from south-west Spain. Bot. Linn. Soc., 115: 133-143.

QUINLIVAN, B.J. -1961- The effect of constant and fluctuating temperatures on the permeability of hard seeds of some legume species. Aust. J. Agric. Res., 12: 1009-1022.

MEYER, S.E. \& S.G. KITCHEN -1992- Cyclic seed dormancy in the short-lived perennial Penstemon palmeri. J. Ecol., 80: 115-122.
OOSTING, H.J. \& M.E. HUMPHREYS -1940Buried viable seeds in a succesional series of old field and forest soils. Bull. Torrey Bot. Club, 67: 253-273.

SOKAL, R.R. \& F.J. ROHLF -1979-Biometría. Blume.

Aceptado para su publicación en Octubre de 1994

Dirección de los autores. Departamento de Biología Vegetal y Ecología. Universidad de Sevilla. Apartado de Correos 1095, E-41080 Sevilla. España. 